\title{
Isolated dysarthria due to extracerebellar lacunar stroke: a central monoparesis of the tongue
}

\author{
Peter P Urban, Susanne Wicht, Hanns Ch Hopf, Susanne Fleischer, Otmar Nickel
}

Department of
Neurology
P P Urban
S Wicht
H Ch Hopf

Department of Communication Disorders

$S$ Fleischer

Department of Nuclear Medicine, University of Mainz, Germany

O Nickel

Correspondence to: Dr Peter P Urban, Department of Neurology, University of Mainz, Langenbeckstrasse 1, D 55101 Mainz, Germany. Telephone 00496131 175162; fax 00496131 173271 .

Received 15 June 1998 and in revised form 29 September 1998 Accepted 16 October 1998

\begin{abstract}
Objectives-The pathophysiology of dysarthria can preferentially be studied in patients with the rare lacunar stroke syndrome of "isolated dysarthria".

Methods- A single study was carried out on seven consecutive patients with sudden onset of isolated dysarthria due to single ischaemic lesion. The localisation of the lesion was identified using MRI. The corticolingual, cortico-orofacial, and corticospinal tract functions were investigated using transcranial magnetic stimulation. Corticopontocerebellar tract function was assessed using ${ }^{99 \mathrm{~m}}$ Tc hexamethylpropylene amine oxime-single photon emission computerised tomography (HMPAO-SPECT) in six patients. Sensory functions were evaluated clinically and by somatosensory evoked potentials.

Results-Brain MRI showed the lesions to be located in the corona radiata $(n=4)$ and the internal capsule $(n=2)$. No morphological lesion was identified in one patient. Corticolingual tract function was impaired in all patients. In four patients with additional cortico-orofacial tract dysfunction, dysarthria did not differ from that in patients with isolated corticolingual tract dysfunction. Corticospinal tract functions were normal in all patients. HMPAO-SPECT showed no cerebellar diaschisis, suggesting unimpaired corticopontocerebellar tract function. Sensory functions were not affected.
\end{abstract}

Conclusion-Interruption of the corticolingual pathways to the tongue is crucial in the pathogenesis of isolated dysarthria after extracerebellar lacunar stroke. (F Neurol Neurosurg Psychiatry 1999;66:495-501)

Keywords: dysarthria; lacunar stroke; corticobulbar tract; magnetic evoked potentials; SPECT

Dysarthria is common in cerebral lesions of different origin and location. However, even in patients in whom the site of the lesion was

Table 1 Clinical and MRI data from patients with isolated dysarthria due to lacunar stroke

\begin{tabular}{llllll}
\hline Patient & Sex/age & Lesion/diameter $(\mathrm{cm})$ & FP & LP & Risk factors \\
\hline 1 & $\mathrm{M} / 55$ & R corona radiata/1.5 & + & + & SM \\
2 & $\mathrm{~F} / 71$ & L internal capsule/0.5 & + & - & HT \\
3 & $\mathrm{M} / 50$ & L corona radiata/1.0 & - & - & HT, SM, HL \\
4 & $\mathrm{~F} / 50$ & L internal capsule $/ 1.0$ & + & + & HT \\
5 & $\mathrm{M} / 48$ & no lesion/-- & + & - & HT, DM \\
6 & $\mathrm{~F} / 76$ & L corona radiata/1.5 & - & + & HT, DM, HL \\
7 & $\mathrm{M} / 62$ & L-corona radiata/1.0 & + & - & HL, DM, SM \\
\hline
\end{tabular}

$\mathrm{FP}=$ facial paresis, $\mathrm{LP}=$ lingual paresis, $\mathrm{R}=$ right, $\mathrm{L}=$ left, $\mathrm{SM}=$ cigarette smoking, $\mathrm{HT}=$ arterial hypertension, $\mathrm{HL}=$ hyperlipidaemia, $\mathrm{DM}=$ diabetes mellitus. apparent from imaging studies, a definite conclusion on the involvement or sparing of individual fibre tracts could not be reached due to the close proximity and varying location of the tracts so that the nature of dysarthria has never been clear. Dysarthria due to stroke is most often associated with other neurological deficits such as hemiparesis, hemiataxia, clumsiness of one hand, central facial paresis, and tongue deviation. ${ }^{1}$ To exclude interferences due to accompanying neurological deficits, we selected patients with isolated dysarthria due to a small singular ischaemic lesion. This lacunar syndrome ${ }^{2}$ is exceedingly rare, as shown in a recent consecutive series of 227 patients with lacunar infarction in whom isolated dysarthria was noted in only $0.4 \% .{ }^{3}$ In seven patients with isolated dysarthria we functionally tested the relevance of major pathways involved in speech production.

\section{Patients and methods}

We report on seven consecutive patients with sudden onset of dysarthria in the absence of other previous or current neurological signs or symptoms. The clinical findings in each patient, including the risk factors for stroke, are summarised in table 1. Dysarthria was diagnosed on the basis of auditory-perceptual presentation and confirmed by two experienced speech therapists. Speech function was assessed using a neurophonetic test battery (modified from Ziegler $e t a l^{4}$ ). Articulation was evaluated on the basis of various samples - that is, spontaneous speech, repetition of sentences and words, reading a short story, and rapid iteration of syllables $(/ \mathrm{pa} /, / \mathrm{ta} /, / \mathrm{ka} /)$. The examination of laryngeal function included laryngoscopy, stroboscopy, and perceptual examination of voice quality, voice stability, pitch, and loudness. Sustained realisation of vowels and fricatives and repetition of sentences of increasing length provided information on respiratory support. Speech tempo was measured based on the syllable repetition rate per second using a sound spectrograph (CSL 4300; Kay Elemetrics Corp, Pine Brook, NJ, USA).

The localisation of the lesion was identified by MRI. Horizontal and coronal or sagittal planes were obtained with conventional spin echo techniques using a 0.5 or 1.5 Tesla tomograph (Philips T5/ACS). All images were T1 and T2 weighted and gadolinium enhanced. Slice thickness was $5 \mathrm{~mm}$ without gaps.

The atlases published by Matsui and Hirano ${ }^{5}$ and Nieuwenhuys et $a l^{6}$ were used as anatomical references.

The corticolingual projections were examined by activating the tongue muscles using transcranial magnetic stimulation (TMS) and 
Table 2 Results of transcranial magnetic stimulation in patients with isolated dysarthria due to lacunar stroke.

\begin{tabular}{|c|c|c|c|c|c|c|c|}
\hline \multicolumn{2}{|c|}{ Stimulation site } & \multicolumn{2}{|l|}{ Cortex $L$} & \multicolumn{2}{|l|}{ Cortex $R$} & \multicolumn{2}{|l|}{$L$ Cortex $R$} \\
\hline \multicolumn{2}{|c|}{ Recording site } & \multicolumn{2}{|l|}{$R$ tongue $L$} & \multicolumn{2}{|c|}{$R$ tongue $L$} & \multicolumn{2}{|c|}{$R$ buccinator $L$} \\
\hline Patient & Affected hemisphere & $T C T$ & & $T C T$ & & $T C T$ & \\
\hline 1 & $\mathrm{R}$ & 8.4 & 8.5 & --- & --- & 10.3 & 9.7 \\
\hline 2 & $\mathrm{~L}$ & -- & -- & 9.9 & 10.4 & 10.7 & -- \\
\hline 3 & $\mathrm{~L}$ & $11.9^{\star}$ & $17.3^{\star}$ & 8.9 & 10.0 & 9.7 & 11.1 \\
\hline 4 & $\mathrm{~L}$ & $11.4^{\star}$ & $15.9^{\star}$ & 11.2 & 10.8 & 11.4 & 10.5 \\
\hline 5 & No lesion & --- & --- & 8.7 & 8.7 & --- & 10.0 \\
\hline 6 & $\mathrm{~L}$ & --- & --- & 8.6 & 8.4 & $14.9^{\star}$ & 9.0 \\
\hline 7 & $\mathrm{~L}$ & --- & -- & 8.5 & 9.6 & -- & 10.2 \\
\hline \multicolumn{2}{|c|}{ Controls mean (SD) } & $8.9(0.8)$ & $8.9(0.8)$ & $8.8(1.2)$ & $8.7(1.0)$ & $10.3(1.0)$ & $9.8(1.0)$ \\
\hline \multicolumn{2}{|c|}{ Upper limit (mean+2.5 SD) } & 10.9 & 10.9 & 11.8 & 11.2 & 12.8 & 12.3 \\
\hline
\end{tabular}

Total conduction times (TCT) for the corticolingual (tongue) and corticoorofacial (buccinator muscle) projections (see methods). $\mathrm{R}=$ right, $\mathrm{L}=$ left. Abnormal results are indicated by --- (no response) and * (delayed response- - that is, TCT>upper limit for control subjects, defined as mean $+2.5 \mathrm{SD}$ )

recording the compound muscle action potentials (CMAPs) at either half of the tongue. Two pairs of $\mathrm{Ag} / \mathrm{AgCl}$ surface disc electrodes at an interelectrode distance of $18 \mathrm{~mm}$ were mounted on a spoon shaped metacrylate device adapted to the oral cavity. The electrodes were placed above the lateral dorsum of the tongue. Slight contraction of the tongue muscles was achieved by gently pressing the dorsum of the tongue against the mouthpiece.

The cortico-orofacial projections were investigated by activating the orofacial muscles using TMS and recording the CMAP of the buccinator muscles at either side of the face. We used pairs of $\mathrm{Ag} / \mathrm{AgCl}$ surface disc electrodes embedded at a distance of $18 \mathrm{~mm}$ in a specially designed fork shaped metacrylate device which was adapted to the oral vestibulum. The electrodes were in contact with the insides of the cheeks. Slight contraction of the buccinator muscles was achieved by pursing the lips.

Filter settings for CMAP recordings were 20-2000 Hz. A Magstim 200S (Novametrix, Whitland, Dyfed, UK) and a circular coil (mean diameter $9 \mathrm{~cm}$ ) with a peak magnetic field of 2.0 Tesla were used for TMS.

For cortical stimulation the centre of the coil was positioned tangentially, 4-6 cm (tongue) and $1-2 \mathrm{~cm}$ (buccinator muscle) lateral to the vertex, at the vertex (upper limbs) and $4 \mathrm{~cm}$ in front of the vertex (lower limbs). On stimulation of the left (right) hemisphere, side " $\mathrm{A}$ " ("B") was viewed from above. Stimulation strength was increased stepwise during slight preinnervation until stable latencies were achieved. Out of four recorded responses the shortest onset latency (total conduction time, TCT) and largest amplitude (peak to peak) of the CMAP were measured.

Table 3 HMPAO tracer uptake of the cerebellar hemisphere in patients with dysarthria due to extracerebellar lacunar stroke.

\begin{tabular}{lllllll}
\hline Patient & Cerebellar hemisphere & Slice 1 & Slice 2 & Slice 3 & Slice 4 & Slice 5 \\
\hline 1 & 0.98 & 1.02 & 1.00 & 0.95 & 0.99 & 0.96 \\
2 & 0.97 & 0.99 & 0.99 & 0.97 & 0.95 & 0.97 \\
3 & 0.97 & 1.01 & 0.98 & 0.97 & 0.95 & 0.93 \\
4 & N.P. & NP & NP & NP & NP & NP \\
5 & 0.94 & 0.90 & 0.91 & 0.97 & 1.04 & 0.87 \\
6 & 1.02 & 1.02 & 1.01 & 1.03 & 1.03 & 1.03 \\
7 & 0.95 & 0.93 & 0.95 & 0.95 & 0.96 & 0.94 \\
\hline
\end{tabular}

Left/right ratio of total counts for the entire cerebellar hemisphere and for each slice in craniocaudal direction. $\mathrm{NP}=$ not performed. To avoid partial volume effects, the upper and lower slices were not considered (cranial slice 1, caudal slice 5). The normal range (mean $\pm 2.5 \mathrm{SD}$ ) of left/right ratio has been reported as $0.82-1.18$ (Perani et $\mathrm{l}^{14}$ ).
A detailed description of lingual and facial recording techniques and normative data have been published elsewhere. ${ }^{7-10}$

Sensation in the oral cavity was tested with pinprick, touch, two point discrimination, and stereognosis (using stimuli of different shape (a cube, ball, or ring)) as suggested by Ringel and Ewanowski ${ }^{11}$ and Ringel et al..$^{12}$ SSEPs were elicited at the median nerve using a standard technique outlined in the IFCN committee guidelines. ${ }^{13}$

SPECT imaging was performed on six patients of this series. After the patients had rested in a dark and silent room for a period of 20 minutes, $550 \mathrm{MBq}{ }^{99 \mathrm{~m}}$ Tc-hexamethylamineoxime (HMPAO) were administered intravenously. After another 10 minute period the patient was placed in the supine position with the head fixed in an adjustable head holder and the images were obtained. Special care was taken to avoid head tilting. A double head rotating gamma camera (Picker, Prism 2000) interfaced to a computer (Picker, Odyssey) with a $20 \%$ symmetric energy window centred on the $140 \mathrm{keV}$ peak was used. A total of 120 $20 \mathrm{~s}$ images were obtained over a 360 degree circular revolution (step and shoot paradigm), using a low energy, high resolution parallel hole collimator. The average radius of rotation was $17 \mathrm{~cm}$. The resolution of the system was 12 $\mathrm{mm}$ and expressed as full width at half maximum at the centre of the field of view and at a depth of $15 \mathrm{~cm}$ from the camera face. A total of 2-4 million total counts were collected over a period of 25 minutes. The images were acquired in a $64 \times 64$ matrix. One pixel (5.8 $\mathrm{mm}$ ) thick transverse oblique slices were reconstructed in parallel to the orbitomeatal line using a low pass filter (Butterworth). Attenuation correction was performed (Chang algorithm) with an attenuation coefficient of $0.13 / \mathrm{cm}$. To compare the tracer uptake between both cerebellar hemispheres from each slice of the cerebellum, a region of interest (ROI) was drawn around the contour of one hemisphere. On mirroring the ROI onto the other hemisphere with the midline serving as the axis, count rates could be acquired within identical regions. To avoid partial volume effects, the upper and the lower slices of the cerebellum were not considered for analysis. ROIs were drawn for each slice at the transverse oblique level to allow better comparison with 


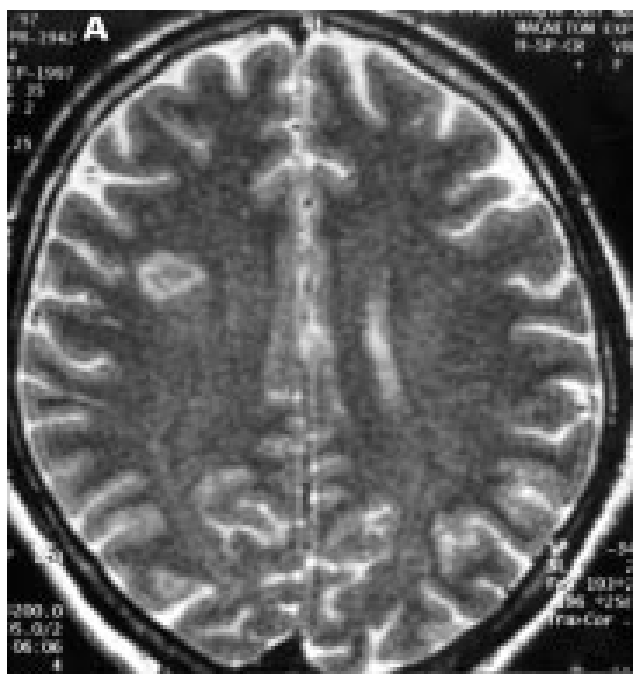

$\stackrel{5 \mathrm{~ms}}{0.5}$

$\mathrm{R}$
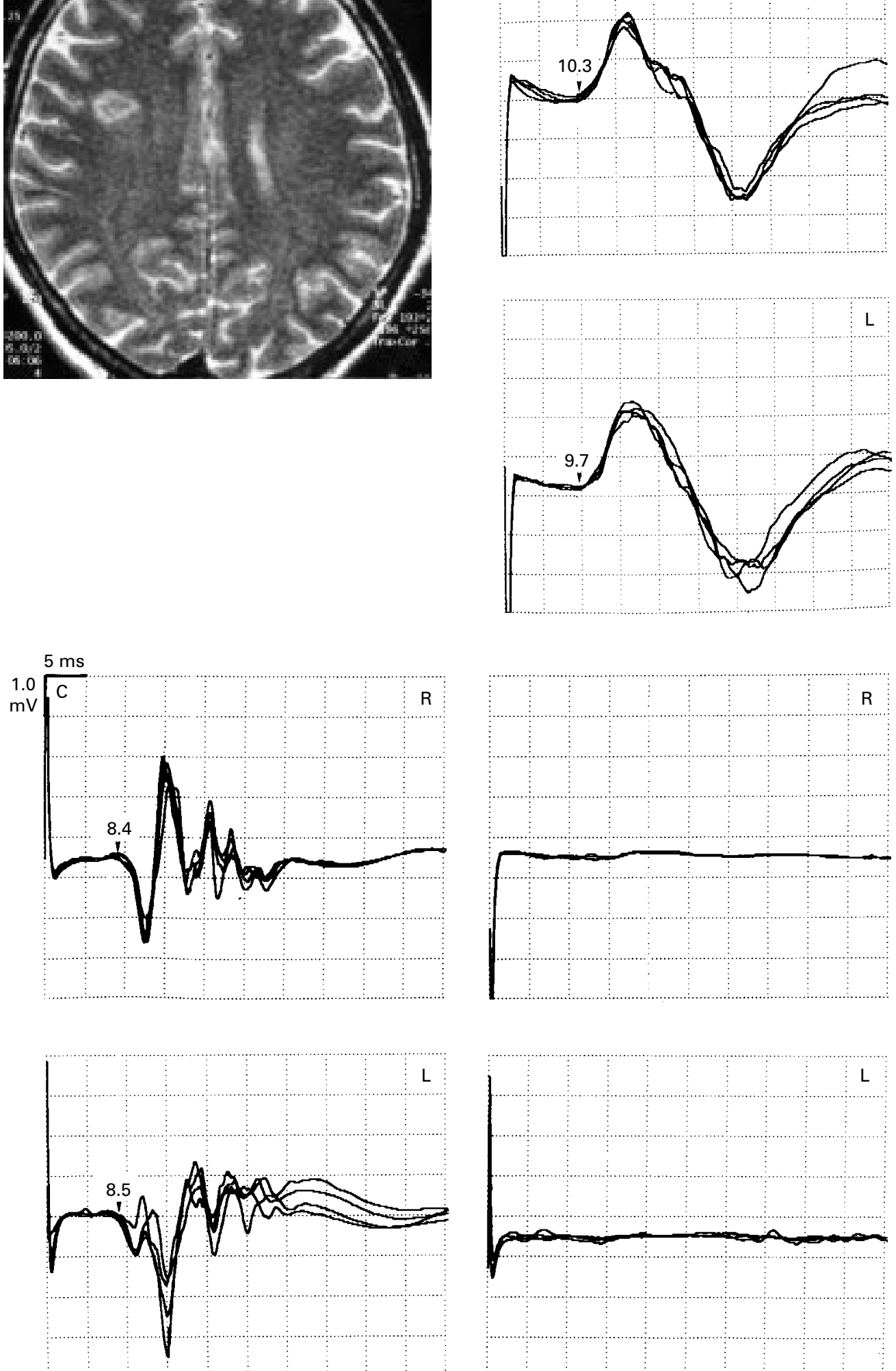

Figure 1 (A) MRI lacunar infarct in the right corona radiata (left side of the figure, patient 1). (B) MEP to the orofacial muscles, showing normal contralateral responses after bilateral cortical stimulation. (C) MEP to the tongue muscles, showing absent responses at both halves of the tongue after magnetic stimulation over the affected right hemisphere muscles, showing absent
(right side of the figure). 

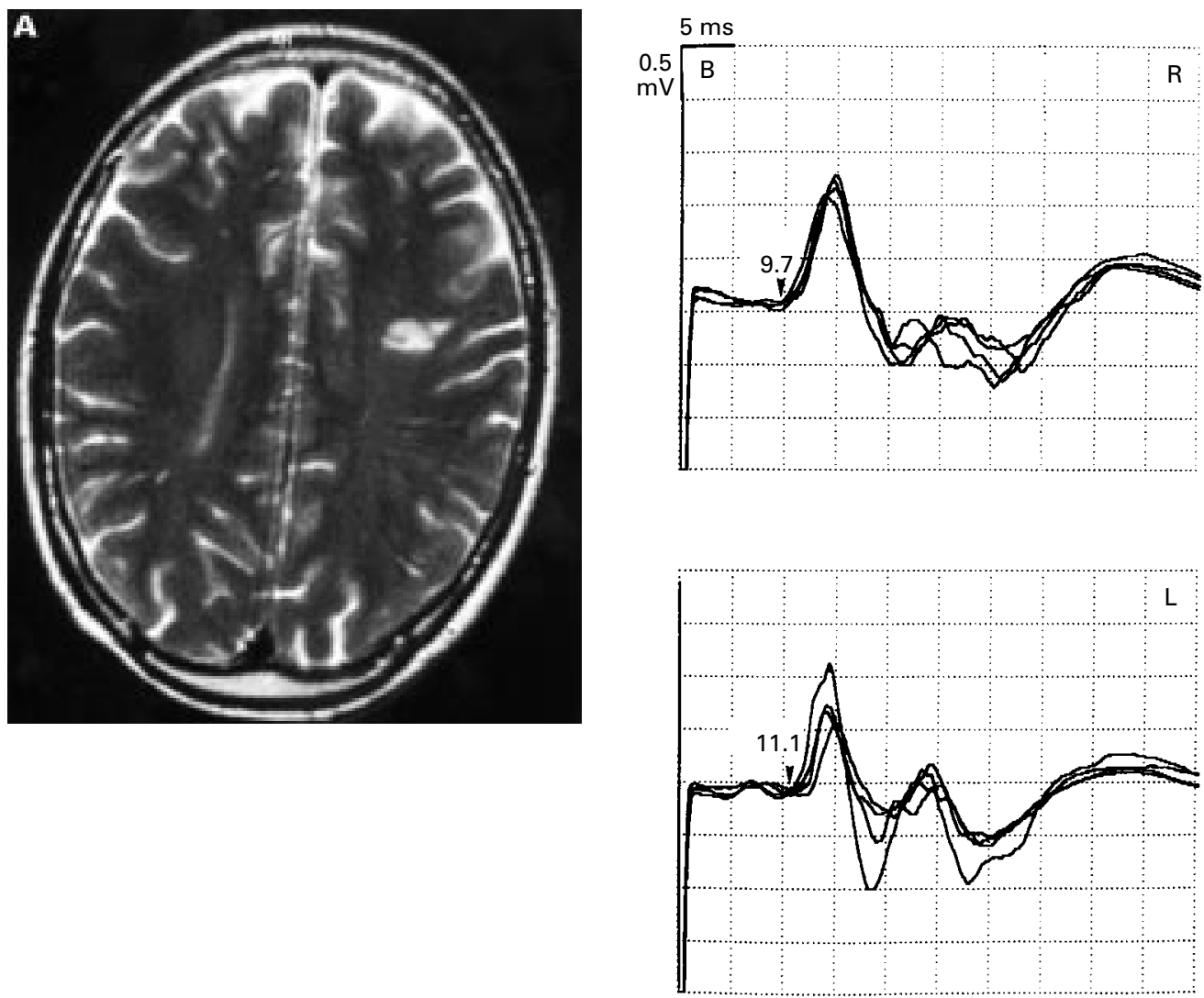

$\sqrt[5.5]{\mathrm{mV}}$

$\mathrm{R}$
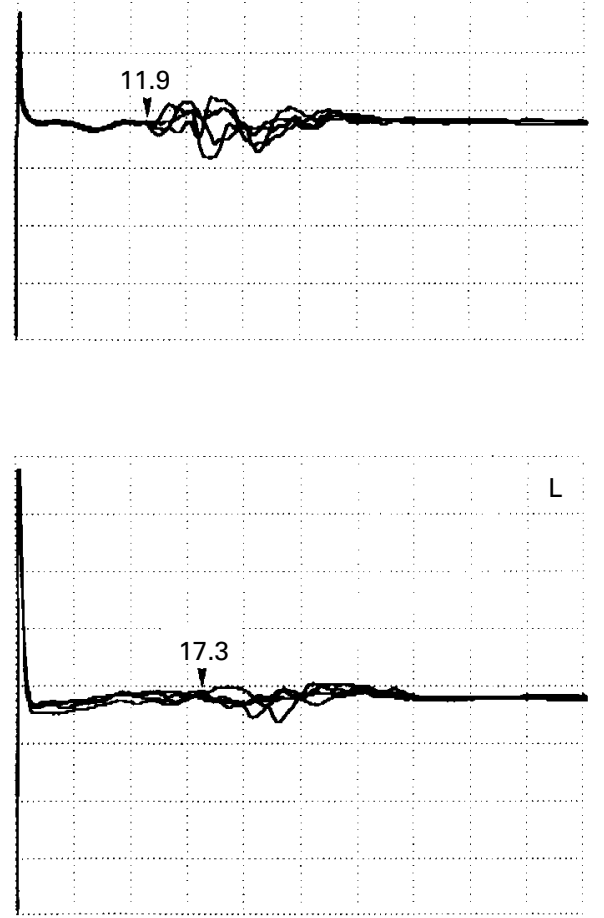
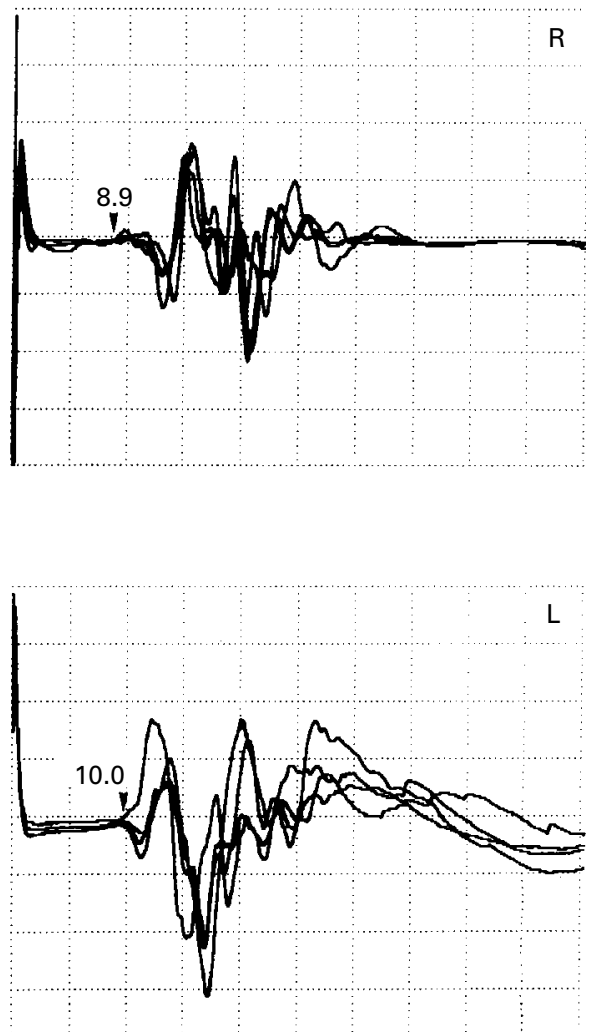

Figure 2 (A) MRI lacunar infarct in the left corona radiata (right side of the figure, patient 3). (B) MEP to the orofacial muscles, showing normal contralateral responses after bilateral cortical stimulation. (C) MEP to the tongue muscles, showing delayed responses at both halves of the tongue $(L>R)$ after magnetic stimulation over the affected left hemisphere (left side of the figure). 
MRI. The left/right ratio was calculated for the count rate of each slice and as a global count rate for the entire cerebellar hemispheres (adding the count rate of all slices). The global left/right cerebellar asymmetry in normal subjects $(n=9)$ was established at a mean of 1.00 (SD 0.07) and a range from 0.82 to 1.18 (mean $\pm 2.5 \mathrm{SD}){ }^{14}$ For ethical reasons we therefore abstained from establishing normal values in the present study.

Informed consent for this study was obtained from all participants and the study was approved by the local ethics committee (Landesärztekammer Rheinland-Pfalz).

\section{Results}

All patients presented with sudden onset of speech difficulties. Dysarthria was characterised by slurring with imprecise articulation and the patients reported a "thick" tongue. Articulatory movements and speech rate were mildly slowed, showing a mean syllable repetition rate of 4.7 syllables/s (normal rate; 6 syllables/s). Modulation of pitch and intensity were reduced. Scanning, explosive, or dysprosodic speech was absent. The degree of dysarthria was mild to moderate in most cases; no patient had unintelligible speech. The voice was breathy, sometimes pressed, and slightly hoarse. Laryngoscopy showed normal vocal fold motility.

The neurological examination showed no deficits unrelated to dysarthria: three patients (patients 1, 4, 6) showed slight tongue deviation and five patients (patients $1,2,4,5$, 7) demonstrated mild facial paresis of the central type. None of the patients showed palatal weakness. However, only one patient showed no clinical deficit beside dysarthria. Sensory function of the oral cavity was unimpaired. Median nerve SSEPs were normal in all patients. Dysarthria cleared within 2 weeks to 6 months (median: 1.2 months).

In all patients TMS of the lesion side (above the affected hemisphere) showed absent (patients $1,2,5,6,7)$ or delayed (patients 3,4 ) corticolingual responses at both halves of the tongue (table 2). TMS evoked orofacial responses contralateral to the lesion side were absent in three patients (patients 2, 5, 7), delayed in one (patient 6) and present in three. TMS of the peripheral hypoglossal and facial nerve elicited biphasic CMAPs at PCT within the normal range in all patients showing normal peripheral impulse conduction. The corticospinal projections to the upper and lower limbs were normal in all patients.

HMPAO-SPECT (table 3) performed in six of seven patients showed no significant side differences in global tracer uptake between the cerebellar hemispheres compared with control data. ${ }^{14}$ For the left/right ratio of single slices, there was no focal reduction in tracer uptake within the cerebellar hemisphere.

Brain MRI showed single lesions in the corona radiata $(n=4)$ and genu and posterior limb of the internal capsule $(n=2)$ without other lacunar infarctions (table 1). In patient 5 no morphological lesion was identified.
No differences in dysarthria were noted with regard to the location and size of the lesion or the side of the affected hemisphere. We found no correlation between the neurophysiological abnormalities (absence $v$ delay of responses, isolated corticolingual $v$ combined corticolingual and cortico-orofacial involvement) and the severity or auditive presentation of dysarthria.

\section{Discussion}

Dysarthria in association with facial and lingual hemiparesis due to lesions established at necropsy in the genu of the internal capsule were described as early as about 100 years ago $^{15-17}$ (for a historical review see Bogousslavsky and $\operatorname{Regli}^{18}$ ). However, due to the close proximity and varying location of fibre tracts no definite conclusions concerning the nature of dysarthria could be achieved.

The pathophysiology of dysarthria may be derived from focal brain lesions, especially in lacunar stroke, compared with large, widespread, or multifocal disorders such as brain tumour (rarely limited to one anatomical structure; oedema, and microscopic tumour spread make an anatomicofunctional correlation more difficult than for discrete infarctions), haemorrhage (leads to mass effects and dissection of blood in neighbouring structures), and degenerative pathological conditions - for example, Parkinson's and Huntington's disease. The last disorders may also be associated with dysarthria although the fact that several central nervous system structures are involved makes it difficult here to identify the fibre tracts attributable to dysarthria. Unilocal lacunar infarcts thus represent a disorder allowing the correlation of clinical signs and symptoms with anatomical structures and functional testing. We therefore investigated seven patients with isolated dysarthria due to lacunar stroke over a three year period.

Impaired articulation is one of the most prominent features of dysarthria. As the tongue and orofacial muscles are the most important articulators ${ }^{1920}$ we investigated the corticolingual and cortico-orofacial pathways using TMS. Corticolingual fibres project bilaterally from either hemisphere to the hypoglossal nuclei $^{821}$ whereas cortico-orofacial fibres project predominantely to the contralateral subnuclei. ${ }^{1021}$ The degree of limb muscle paresis correlates with an increase in latency and decrease in amplitude of the TMS muscle response in ischaemic cerebral lesions. ${ }^{22}{ }^{23}$ These parameters reflect the degree of functional impairment of the fast conducting large diameter pyramidal fibres. ${ }^{24}$ The absence of a response is associated with a more severe lesion than a delayed response. Because amplitudes of the TMS evoked potentials show a wide interindividual variation, ${ }^{25}{ }^{26}$ which also applies to tongue and orofacial muscle responses, ${ }^{8} 10$ only absent or delayed $(>$ mean $+2.5 \mathrm{SD})$ responses were considered as abnormal. Control values were obtained from 43 healthy subjects. ${ }^{9} 10$

The characteristics of dysarthria in our patients were almost identical. Dysarthria was mild to moderate. No differences in dysarthria 
were noted with regard to the location and size of the lesion and the side of the affected hemisphere. The most common features were imprecise articulation, a mildly slowed speech rate, and a slightly monotonous voice. These auditory findings are similar to those reported previously for central motor impairment due to hemispheric infarction. ${ }^{27}{ }^{28}$ The uniformity of speech abnormality found in the described locations is consistent with a common pathophysiological basis.

The common abnormality in all patients with dysarthria was involvement of the corticolingual projections as disclosed by TMS. However, only three (patients $1,4,6$ ) out of seven patients had clinical signs of a tongue movement disorder (fig 1A-C). One patient (patient 3) showed dysarthria without any further clinical deficits. In this patient, TMS of the left motor cortex showed delayed responses at both halves of the tongue due to lacunar infarction in th left corona radiata (fig $2 \mathrm{~A}-\mathrm{C}$ ). In four patients with additional cortico-orofacial tract involvement speech performance was not more significantly or differently disturbed, suggesting that impairment of the cortico-orofacial tract does not necessarily contribute to the development of dysarthria in lacunar stroke. This assumption was recently confirmed in a patient with an isolated cortico-orofacial tract lesion, who did not show dysarthria. ${ }^{29}$

Normal TMS results of the peripheral hypoglossal and facial nerves and the absence of clinical signs at peripheral facial and hypoglossal nerve lesions in our patients indicate that the observed conduction abnormalities after cortical stimulation must be attributed to the central lesions shown by MRI. The lesions were located within the pyramidal tract, ${ }^{56}$ between the lower motor cortex and the genu and the posterior limb of the internal capsule. The left hemisphere (six patients) was more often affected than the right hemisphere (one patient). This confirms previous findings, that dysarthria is not restricted to left sided lesions only, but to a lesser degree also occurs in right sided lesions. ${ }^{30}{ }^{31}$ In one patient (patient 5) TMS showed corticofacial and corticolingual tract involvement although MRI did not identify a morphological lesion. However, negative MRI findings in lacunar infarction are not uncommon, ${ }^{32-34}$ reflecting the still limited ability of MRI in detecting small lacunar lesions.

The detection of a larger number of subclinical corticolingual tract lesions than of subclinical cortico-orofacial tract lesions by TMS may be due to the bilateral symmetric tongue innervation which clinically masked the sequels of a lesion in one hemisphere, whereas the predominantly unilateral (contralateral) innervation of the lower facial muscles was more often associated with clinically apparent paresis. The not infrequently found coincidence of corticolingual and corticofacial tract involvement likely results from the close proximity of both fibre tracts along their entire course from motor cortex to brainstem..$^{213}$ Most ischaemic lesions therefore affect not only the corticolingual pathway. Both factors may explain the clinical finding that dysarthria due to the unilateral vascular lesion of one cerebral hemisphere is most often associated with the clinical finding of facial, but not of unilateral tongue weakness. ${ }^{3036}$

Sensory deficits are not the only factors related to dysarthria, ${ }^{37}$ which is confirmed by the fact that the intraoral sensory functions and median nerve SSEPs were undisturbed in all our patients.

Because dysarthria has been attributed to corticopontocerebellar tract involvement, ${ }^{38} 39$ we performed HMPAO-SPECT, which is associated with a reduced tracer uptake of one cerebellar hemisphere (cerebellar diaschisis) in the presence of corticopontocerebellar tract dysfunction. $^{40}{ }^{41}$ In our patients HMPAOSPECT studies showed no cerebellar diaschisis, indicating that corticopontocerebellar tract function was unimpaired, although partial corticopontocerebellar tract dysfunction cannot completely be excluded due to the limited spatial resolution of HMPAO-SPECT.

In conclusion, functional testing of the major pathways involved in articulation disclosed unilateral interruption of the corticolingual projections in all patients with isolated dysarthria due to lacunar stroke outside the cerebellum. As the hypoglossal system is responsible for the entire motor innervation to the tongue muscles, whose precise and highly coordinate interactions are required for the production of different sounds, ${ }^{42}{ }^{43}$ we conclude that impairment of the central lingual motor subsystem is a major factor accountable for imprecise articulation in dysarthric speakers. Due to the somatotopically arranged pyramidal tract fibres, partial motor deficits may occur with lacunar lesions in the corona radiata and internal capsule leading to a central monoparesis of the face, ${ }^{44}{ }^{45}$ upper limb, ${ }^{4647}$ and lower limb. ${ }^{48}$ We have now shown that a central monoparesis of the tongue may also occur, clinically presenting as isolated dysarthria.

We thank S Hartmann and N Sahler for performing speech examinations and speech therapy. We thank the DFG (Deutsche Forschungsgemeinschaft) for financial support of this project (Ur 37/2-1).

1 Ichikawa K, Kageyama Y. Clinical anatomic study of pure dysarthria. Stroke 1991;22:809-12.

2 Fisher CM. Lacunar strokes and infarcts: a review. Neurology 1987;32:871-6.

3 Arboix A, Marti-Vilalta JL, Garcia JH. Clinical study of 227 patients with lacunar infarcts. Stroke 1990,21:842-7.

4 Ziegler W, Hartmann E, Hoole P, et al. Entwicklung von diagnostischen Standards und von Therapieleitlinien für zentrale Stimm- und Sprechstörungen (Dysarthrophonien). zentrale Stimm- und Sprechstörungen (Dysarthrophonien).
München: Forschungsberichte der Gesellschaft für München: Forschungsberichte der Gesel

5 Matsui T, Hirano H. An atlas of the human brain for compuMatsui T, Hirano H. An atlas of the human b.
terized tomography. Stuttgart: Fischer, 1978.

6 Nieuwenhuys R, Voogd J, van Huijzen C. The human central nervous system. Berlin: Springer, 1988.

7 Urban PP, Hopf HC, Zorowka PG, et al. Dysarthria and lacunar stroke: pathophysiologic aspects. Neurology 1996; 47:1135-41

8 Urban PP, Hopf HC, Connemann B, et al. The course of cortico-hypoglossal projections in the human brain-stem. Functional testing using transcranial magnetic stimulation. Brain 1996;119:1031-8.

9 Urban PP, Hopf HC, Connemann B, et al. Technical considerations of electromyographic tongue muscle recordings using transcranial magnetic stimulation [letter]. Brain 1997;120:1911-4.

10 Urban PP, Beer S, Hopf HC. Cortico-bulbar fibres to orofacial muscles: recordings with enoral surface electrodes. Electroencephalogr Clin Neurophysiol 1997;105:8-14.

11 Ringel RL, Ewanowski SJ. Oral perception: 1.Two-point Ringel RL, Ewanowski SJ. Oral perception: 1.
discrimination. $\mathcal{I}$ Speech Hear Res 1965;8:389-98. 
12 Ringel RL, Burk KW, Scott CM. Tactile perception: form discrimination in the mouth. Br f Disord Commun 1968;3: discrim $150-5$.

13 Nuwer MR Aminoff M, Desmedt J, et al. IFCN recommended standards for short latency somatosensory evoked potentials. Report of an IFCN commitee. Electroencephalogr Clinical Neurophysiol 1994;91:6-11.

14 Perani D, Lucignani G, Pantano P, et al. Cerebella diaschisis in pontine ischemia. A case report with single photon emission computerized tomography. $\mathcal{F}$ Cereb Blood Flow Metab 1987;7:127-31.

15 Etienne G. Monoplégie faciale et deviation conjugée de la face et des yeux D'origine capsulaire. Presse Méd 1896;4:657-8.

16 Daland J. A case of dysphagia and dysphasia resulting from a lesion in the internal capsule. I Nerv Ment Dis 1897;1:614-9.

17 Dejerine J, Dejerine-Klumpke H. Anatomie des centres nerveaux. Vol 2. Paris: J Rueff, 1901.

18 Bogousslavsky J, Regli F. Capsular genu syndrome. Neurology 1990;40:1499-502.

19 Harris KS. Physiological aspects of speech production. Status report on speech research, SR-48. New Haven, CU: Haskins Laboratories, 1976.

20 Langmore SE, Lehman ME. Physiologic deficits in the orofacial system underlying dysarthria in amyotrophic latera sclerosis. F Speech Hear Res 1994;37:28-37.

21 Brodal A. Neurological anatomy. New York: Oxford University Press, 1981:448-577.

22 Abbruzzese G, Morena M, Dall'Agata D, et al. Motor evoked potentials (MEPs) in lacunar syndrome. Electroencephalogr Clin Neurophysiol 1991;81:202-8.

23 Ferbert A, Vielhaber S, Meincke U, et al. Transcranial magnetic stimulation in pontine infarction: correlation to degree of paresis. F Neurol Neurosurg Psychiatry 1992;55: 294-9.

24 Eisen AA, Shtybel W. AAEM minimonograph No 35: clinical experiences with transcranial magnetic stimulation. Muscle Nerve 1990;13:995-1011.

25 Amassian VE, Cracco RQ, Maccabee PJ. Focal stimulation of human cerebral cortex with magnetic coil: a comparison with electrical stimulation. Electroencephalogr Clin Neurowith electrical stimulation

26 Eisen AA, Siejka S, Schulzer M, et al. Age-dependent decline in motor evoked potentials (MEP) amplitude: with a comment on changes in Parkinson's disease. Electroencephalogr Clin Neurophysiol 1990;81:209-15.

27 Ropper AH. Severe dysarthria with right hemisphere stroke. Neurology 1987;37:1061-3.

28 Hartman DE, Abbs JH. Dysarthria associated with focal unilateral upper motor neuron lesion. Eur f Disord Commun 1992;27:187-96.

29 Urban PP, Wicht S, Marx J, et al. Isolated voluntary facial paresis due to pontine ischemia. Neurology 1998;50:185962.
30 Melo TP, Bogousslavsky J, Van Melle G, et al. Pure motor stroke: a reappraisal. Neurology 1992:42:789-98.

31 Urban PP, Hopf HC, Fleischer S, et al. Impaired cortico-bulbar tract function in dysarthria due to hemispheric stroke. Brain 1997;120:1077-84.

32 Rothrock JF, Lyden PD, Hesselink JR, et al. Brain magnetic resonance imaging in the evaluation of lacunar stroke. Stroke 1987;18:781-6.

33 Arboix A, Marti-Vilalta JL, Pujal J, et al. Lacunar cerebral infarct and nuclear magnetic resonance. Eur Neurol 1990b; 30:47-51.

34 Alberts MJ, Faulstich ME, Gray L. Stroke with negative brain magnetic resonance imaging. Stroke 1992;23:663-7.

35 Foerster O. Motorische Felder und Bahnen. In: Foerster O, Bumke O, eds. Handbuch der Neurologie. Band 6. Berlin: Springer, 1936:1-357.

36 Willoughby EW, Anderson NE. Lower cranial nerve motor function in unilateral vascular lesions of the cerebral hemisphere. Br Med f 1984;289:791-794.

37 Ringel RL, Steer MD. Some effects of tactile and auditory alterations on speech output. I Speech Hear Res 1963;6: 369-378.

38 Luchsinger R, Arnold GE. Handbuch der Stimm-und Sprachheilkunde. Wien, New York: Springer, 1970.

39 Ozaki I, Baba M, Narita S, et al. Pure dysarthria due to anterior internal capsule and/or corona radiata infarction: a report of five cases. F Neurol Neurosurg Psychiatry 1986;49: 1435-1437.

40 Bowler JV, Wade JPH. Ipsilateral cerebellar diaschisis following pontine infarction. Cerebrovasc Dis 1991;1:58-60.

41 Fazekas F, Payer F, Valetitsch H, et al. Brain stem infarction and diaschisis. A SPECT cerebral perfusion study. Stroke 1993;24:1162-6.

42 MacNeilage PF, Shales GN. An electromyographic study of the tongue during vowel production. I Speech Hear Res 1964;7:209-32.

43 Bole CT, Lessler MA. Electromyography of the genioglossus in man. F Appl Physiol 1966;21:1695-8.

44 Huang $\mathrm{C}$, Broe G. Isolated facial palsy: a new lacunar syndrome. F Neurol Neurosurg Psychiatry 1984;47:84-6.

45 Kim JS. Pure dysarthria, isolated facial paresis, or dysarthria-facial paresis syndrome. Stroke 1994;25:19948.

46 Benett AH, Campbell CM. Case of brachial monoplegia, due to lesion of the internal capsule. Brain 1885;8:78-84.

47 Takamatsu N, Yamanouchi H, Yamada H. Lacune in the white matter revealed by MRI in a case of pure motor monoparesis. Clin Neurol 1990;30:898-900.

48 Donnan GA, Tress BH, Bladin PF. A prospective study of acunar infarction using computerized tomography. Neurology 1982;32:49-56. 